\title{
Short Communication \\ Membranous location of EGFR immunostaining is associated with good prognosis in renal cell carcinoma
}

\author{
JP Kallio*,I, P Hirvikoski ${ }^{2}$, H Helin ${ }^{3}$, P Kellokumpu-Lehtinen ${ }^{4,5}$, T Luukkaala ${ }^{6}$, TLJ Tammela ${ }^{1,5}$ and \\ PM Martikainen ${ }^{3,5}$
}

'Department of Urology, Tampere University Hospital, PO Box 2000, FIN-3352I Tampere, Finland; ²Department of Pathology, Oulu University Hospital, PO Box 50, FIN-90029 OYS, Oulu, Finland; ${ }^{3}$ Department of Pathology, Tampere University Hospital, PO Box 2000, FIN-3352I Tampere, Finland; ${ }^{4}$ Department of Oncology, Tampere University Hospital, PO Box 2000, FIN-3352I Tampere, Finland; ${ }^{5}$ Medical School, University of Tampere,

FIN-3352I Tampere, Finland; and ${ }^{6}$ School of Public Health, University of Tampere, FIN-330I 4 Tampere, Finland

Epidermal growth factor receptor (EGFR) is a key factor in tumorigenesis. The association between EGFR expression and prognosis in renal cell carcinoma (RCC) is not clear. In our study of 134 RCCs, the cellular location of immunostaining was evaluated and patients with EGFR-positive tumours with prominent membranous staining had a good prognosis. Their overall survival was significantly longer $(P=0.004)$ than that of patients with either EGFR-negative tumours or with mainly cytoplasmic staining. However, further studies on the different EGFR expression patterns in RCC are needed to clarify their role in the progression of the disease. British Journal of Cancer (2003) 89, 1266- 1269. doi:I0.1038/sj.bjc.660 I24I www.bjcancer.com

(c) 2003 Cancer Research UK

Keywords: renal cell carcinoma; epidermal growth factor receptor; prognosis

The epidermal growth factor receptor (EGFR) family has been found to play a central role in tumour progression. Ligand binding to the EGFR, receptor dimerisation and the activation of downstream signalling pathways are molecular events involved in tumorigenesis (Kim and Muller, 1999; Carpenter, 2000. High expression of EGFR is considered to be an unfavourable prognostic factor in patients with a variety of tumours (Lieberman et al, 1985; Slamon et al, 1987; Neal et al, 1990), including renal cell carcinoma (RCC) (Uhlman et al, 1995; Yoshida et al, 1997). However, there are also studies on RCC reporting no association between EGFR and prognosis (Hofmockel et al, 1997). In these studies, in addition to ligand binding and immunohistochemical methods (Yoshida et al, 1997), also Northern blot (Sargent et al, 1989) and Southern blot (Gomella et al, 1989) analyses have been used. In the present study, we examined the association between the location of the EGFR immunostaining and prognosis in RCC.

\section{MATERIALS AND METHODS}

\section{Patients}

Our study population consisted of 134 consecutive patients who underwent radical nephrectomy for RCC between 1995 and 1999 at Tampere University Hospital. The median age of the patients $(83$ men and 51 women) at the time of operation was 64 years (range 35-86 years). Clinical stage was assigned using the TNM Classification of Malignant Tumours (UICC, 1997). The median follow-up time was 40 months, 49 months for survivors and 12

*Correspondence: Dr JP Kallio; E-mail: jukka.kallio@uta.fi

Received 13 February 2003; revised 24 June 2003; accepted 8 July 2003 months for nonsurvivors. During the follow-up time, 40 patients died of RCC and 17 of other causes.

\section{Specimens}

Archival formalin-fixed, paraffin-embedded RCC material was used for the study. All tissue blocks were re-evaluated $(\mathrm{PH})$, and from a representative area for each tumour a 3-mm core was transferred to a multi-tissue block, which was then used for further analysis. All tumours were classified according to Heidelberg classification (Kovacs et al, 1997) and graded according to the Fuhrman system (Fuhrman et al, 1982) by two pathologists (HH, PM).

\section{Immunohistochemical staining}

Paraffin-embedded multi-tissue blocks were cut $4-5 \mu \mathrm{m}$ in thickness and mounted on precoated slides. After deparaffinisation, antigen retrieval was performed by heating the sections in a microwave oven for $2 \times 7 \mathrm{~min}$ in $10 \mathrm{~mm}$ Tris/1 mM EDTA (pH 9.0) buffer, followed by washes with water. A polyclonal rabbit antiEGFr variant III antibody (Zymed Laboratories, Inc., San Francisco, CA, USA) was used for EGFR immunostaining at a concentration of $5 \mu \mathrm{g} \mathrm{ml}^{-1}$. A TechMate ${ }^{\mathrm{TM}} 500$ Plus Immunostainer (DAKO a/s, Glostrup, Denmark) was used for the staining procedure and a ChemMate ${ }^{\mathrm{TM}}$ peroxidase/DAB detection kit (DAKO a/s, Glostrup, Denmark) for visualisation of the antigen antibody complex. Sections were slightly counterstained with haematoxylin.

\section{Evaluation of immunohistochemical staining}

The optimal titre for EGFR staining was defined as the dilution giving clearly identifiable membrane staining and negligible 
background on human placental samples. The intensity of the immunostaining in RCC (scale $0-3$ ) was multiplied by the percentage of cells with positive staining to give a score of 0 300 . Thus scored, the positive placental control (Neal et al, 1990) in our system gave a score of 100 (Figure 1, inset). Five staining patterns were scored: solely $(\mathrm{m})$ or predominantly $(\mathrm{m}>\mathrm{c})$ membranous staining, solely (c) or predominantly $(\mathrm{c}>\mathrm{m})$ cytoplasmic staining or equal $(\mathrm{c}=\mathrm{m})$.

\section{Statistical analysis}

Associations of different staining patterns with other main prognostic parameters were tested using Fisher's exact test. Cox regression analysis was used to test for differences in RCC-specific survival. Both univariate and multivariate analyses were performed. In multivariate analysis, prognostic significance was tested for age at operation time, TNM stage, Fuhrman grade, gender and location of EGFR staining. Deaths from causes other than RCC were considered as censored events. An SPSS software version 11.0 was used for statistical analyses.

\section{Ethics}

The research plan was approved by the Ethical Committee of Tampere University Hospital, and oral informed consent was obtained from every patient.

\section{RESULTS}

Altogether, 69 of the tumours (52\%) were local (T1 -2, N0, M0), 27 (20\%) locally advanced (T3-4, N0, M0) and 38 (28\%) disseminated $(\mathrm{T} 1-4, \mathrm{~N}+/ \mathrm{M}+) ; 122(89 \%)$ of the tumours had conventional type (clear cell), six (5\%) papillary, two (2\%) chromophobe, two (2\%) collecting duct histology. Two of the tumours $(2 \%)$ remained unclassified. By nuclear grading, four
(3\%) of the tumours were classified as grade 1, $59(44 \%)$ as grade 2, $57(43 \%)$ as grade 3 and $14(10 \%)$ as grade 4 .

Epidermal growth factor receptor scores varied between 0 and 300 , mean score was 83 . In all, $73 \%$ of tumours were positive in EGFR immunostaining with cutoff score 20. When placental control (score 100) was used as a cutoff score, the percentage of positive tumours was $49 \%$. Papillary tumours had markedly elevated scores (mean $140 \pm 63$ ) when compared to those with clear cells (mean 79 \pm 69 ). The distribution of EGFR immunostaining was as follows: no staining $23 \%, \mathrm{~m} 3 \%$ (Figure $1 \mathrm{~A}$ ), $\mathrm{m}>\mathrm{c} 11 \%$, $\mathrm{m}=\mathrm{c} 13 \%, \mathrm{c}>\mathrm{m} 25 \%$ and $\mathrm{c} 25 \%$ (Figure 1B). Stratification into three groups was carried out: 1: no staining (23\%), 2: prominent membranous staining $(27 \%$, including cases $\mathrm{m}, \mathrm{m}>\mathrm{c}$ and $\mathrm{m}=\mathrm{c}$ ) and 3: predominantly cytoplasmic staining (50\%, including cases $\mathrm{c}>\mathrm{m}$ and $\mathrm{c}$ ). Papillary tumours all had cytoplasmic staining.

In univariate analysis for survival, predominantly membranous staining (Group 2 in Figure 2) associates with good prognosis, (hazard ratio (HR) $8.0 ; 95 \%$ CI $2.0-33.2 ; P=0.004$ ). When EGFR expression was handled as a continuous numeric factor (score) or divided into two classes (cutoff score 20 or 100), there was no statistically significant association with RCC survival. In the multivariate analysis, TNM stage was a very strong and single significant prognostic indicator (HR 37.0; 95\% CI 8.2-167.5; $P<0.001)$. The membranous immunostaining was not associated with low stages 1-2 $(P=0.091$, Table 1$)$, but there was a statistically significant association with low Fuhrman grades $(P=0.001$, Fisher's exact test, Table 2$)$.

\section{DISCUSSION}

In the present study, we showed the importance of the location of EGFR immunostaining in evaluating the prognosis in an unselected group of patients treated for RCC. The study was carried out on unselected consecutive patients operated for RCC in a prospective manner having a well-defined follow-up schedule.
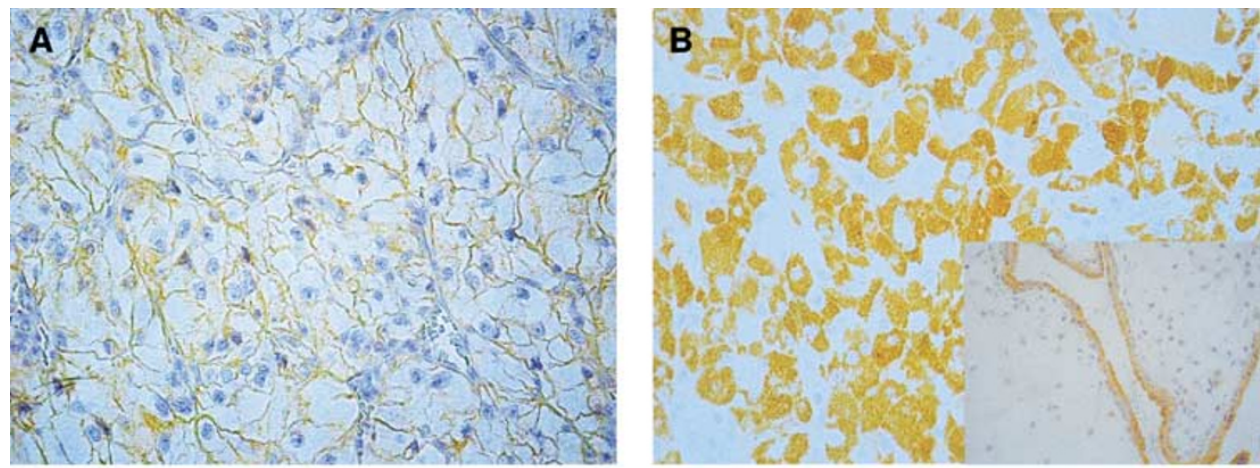


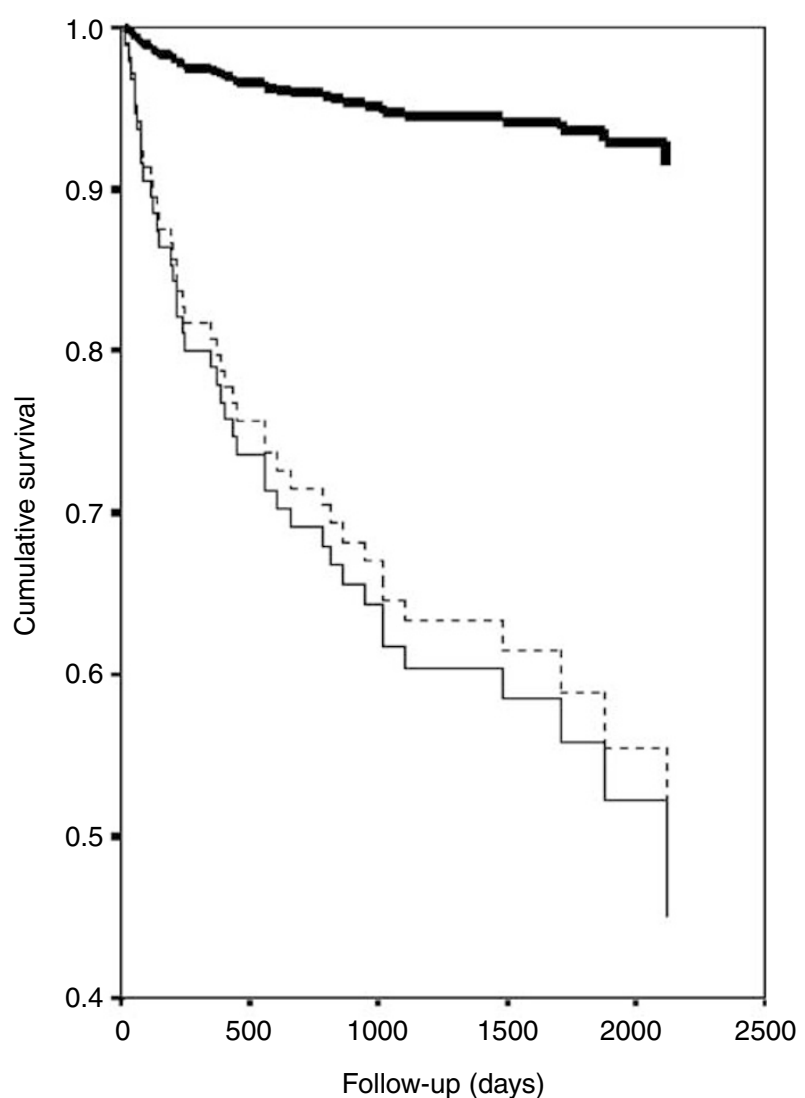

EGFR location

-...- Group $3 \longrightarrow$ Group $2 \longrightarrow$ Group 1

Figure 2 Survival of RCC patients and its correlation with EGFR localisation. Group I: no staining, group 2: prominent membranous staining and group 3: predominantly cytoplasmic staining.

High expression of EGFR has been associated with advanced stage, poor prognosis and high metastatic potential in many human tumours (Lieberman et al, 1985; Slamon et al, 1987; Neal et al, 1990). The association between EGFR expression and prognosis in RCC has not been established. There are several complicating factors: the histological heterogeneity of RCC and the individual properties of different assessment methods. For example, the ligand binding method, which has been held to be a sensitive method for measurement of EGFR, measures functional properties and the technique concentrates on membranous proteins only (Yoshida et al, 1997). Immunohistochemistry, on the other hand, offers a simple and economical means for cellular detection of EGFR and analysing its location in tumour cells. So far, several immunohistochemical studies have shown that positive EGFR staining in RCC is common and is associated with cell proliferation (Moch et al, 1997), but its role as a prognostic factor remains uncertain (Hofmockel et al, 1997). In the immunohistochemical study by Uhlman et al (1995), membranous EGFR expression was associated with high tumour grade, metastatic disease and poor disease-specific survival. In contrast to that, in our study membranotic positivity was associated with good prognosis, while cytoplasmic or negative EGFR staining was not. The explanation for this discrepancy is unclear. However, in the present study, both the intensity and location of EGFR staining in tumour cells were taken
Table I Crosstabulated EGFR immunostaining location classes vs stages

\begin{tabular}{lcccc}
\hline & \multicolumn{4}{c}{ Stage } \\
\cline { 2 - 5 } EGFR staining location & $\mathbf{1}$ & $\mathbf{2}$ & $\mathbf{3}$ & $\mathbf{4}$ \\
\hline No staining & 11 & 4 & 5 & 11 \\
Membranous staining & $20.4 \%$ & $26.7 \%$ & $17.9 \%$ & $29.7 \%$ \\
& 19 & 12 & 11 & 4 \\
Cytoplasmic staining & $35.2 \%$ & $13.3 \%$ & $39.3 \%$ & $10.8 \%$ \\
& 24 & 9 & 12 & 22 \\
& $44.4 \%$ & $60.0 \%$ & $42.9 \%$ & $59.5 \%$ \\
& $100 \%$ & $100 \%$ & $100 \%$ & $100 \%$ \\
\hline
\end{tabular}

No statistical associations, $P=0.09$ I, Fisher's exact test.

Table 2 Crosstabulated EGFR immunostaining location classes vs Fuhrman nuclear grades

\begin{tabular}{lcccc}
\hline & \multicolumn{4}{c}{ Fuhrman nuclear grade } \\
\cline { 2 - 5 } EGFR staining location & $\mathbf{I}$ & $\mathbf{2}$ & $\mathbf{3}$ & $\mathbf{4}$ \\
\hline No staining & 0 & 11 & 15 & 5 \\
& $0.0 \%$ & $18.6 \%$ & $26.3 \%$ & $35.7 \%$ \\
Membranous staining & 3 & 25 & 6 & 2 \\
& $75.0 \%$ & $42.4 \%$ & $10.5 \%$ & $14.3 \%$ \\
Cytoplasmic staining & 1 & 23 & 36 & 7 \\
& $25.0 \%$ & $39.0 \%$ & $63.2 \%$ & $50.0 \%$ \\
& $100 \%$ & $100 \%$ & $100 \%$ & $100 \%$ \\
\hline
\end{tabular}

The membranous immunostaining has a statistically significant association with low Fuhrman grades, $P=0.00$ I, Fisher's exact test

into account when evaluating its associations with prognosis, while in the study by Uhlman et al, there is no description of staining pattern and differences in antibody and staining process may partly explain different results. A similar adverse prognostic role of cytoplasmic EGFR staining demonstrated in our study has also been shown in squamous cell carcinoma of the lung (Piyathilake et al, 2002). The association between poor prognosis and cytoplasmic EGFR staining may be due to changes in ligand-EGFR complex internalisation and activation of associated signalling pathways in the progression of RCC, the theory that Piyathilake et al have further strengthened by cell culture experiments. The localisation pattern of immunostaining has also been shown to have prognostic value with other markers in cancer like bcl-2 expression in malignant melanoma. In studies by Vlaykova et al (2002), a diffuse localisation of bcl-2 expression by immunostaining was associated with better survival than negative or focal expression in malignant melanoma. Similarly, the aberrant cellular location of some adhesion molecules such as alpha-catenin may result in tumour dedifferentiation and aggressive, metastatic phenotype (Hirvikoski et al (1998) in laryngeal carcinoma). In the present study, the presence of immunostaining in the membranes was associated with exceptionally good prognosis. It can be speculated that in RCCs in which EGFR distribution in cell membranes is maintained, the growth is probably still controlled by EGF rather than by activation of new signalling pathways. Thus, the distinction between two different survival groups within the EGFR-positive RCCs, in addition to being a candidate for a simple prognostic marker, opens up the challenging possibility of different molecular targets for drug development for these patient groups. The third group in our study, RCCs with totally negative EGFR staining, may include a group of cancers in which mechanisms other than EGF are responsible for cancer growth and progression. 
In our study, prominent membranous EGFR immunostaining associates with good prognosis in RCC. However, further studies will be needed to clarify the role of the different EGFR patterns in development and progression of RCC.

\section{REFERENCES}

Carpenter G (2000) The EGF receptor: a nexus for trafficking and signalling. BioEssays 22: $697-707$

Fuhrman SA, Lasky LC, Limas C (1982) Prognostic significance of morphologic parameters in renal cell carcinoma. Am J Surg Pathol 6: $655-663$

Gomella LG, Anglard P, Sargent ER, Robertson CN, Kasid A, Linehan WM (1989) Epidermal growth factor receptor gene analysis in renal cell carcinoma. J Urol 143: 191 - 193

Hirvikoski P, Kumpulainen EJ, Virtaniemi JA, Helin HJ, Rantala I, Johansson RT, Juhola M, Kosma V-M (1998) Cytoplasmic accumulation of alpha-catenin is associated with aggressive features in laryngeal squamous-cell carcinoma. Int J Cancer 79: 546-550

Hofmockel G, Riess S, Bassukas ID, Dämmrich J (1997) Epidermal growth factor family and renal cell carcinoma: expression and prognostic impact. Eur Urol 31: 478-484

Kim H, Muller WJ (1999) The role of the epidermal growth factor receptor family in mammary tumorigenesis and metastasis. Exp Cell Res 253: $78-87$

Kovacs G, Akhtar M, Beckwith BJ, Bugert P, Cooper CS, Delahunt B, Eble JN, Fleming S, Ljungberg B, Medeiros LJ, Moch H, Reuter VE, Ritz E, Roos G, Schmidt D, Srigley JR, Storkel S, van den Berg E, Zbar B (1997) The Heidelberg classification of renal cell tumours. J Pathol 182: 131 - 133

Lieberman TA, Nusbaum HR, Razon N, Kris R, Lax I, Soreq H, Waterfield MD, Ullrich A, Schlessinger J (1985) Amplification, enhanced expression of possible rearrangement of EGF receptor gene in primary human brain tumor of glial origin. Nature 313: $144-147$

Moch H, Sauter G, Buchholz N, Gasser TC, Bubendorf L, Waldman FM, Mihatsch MJ (1997) Epidermal growth factor receptor expression is associated with rapid tumor cell proliferation in renal cell carcinoma. Hum Pathol 28: $1255-1259$

\section{ACKNOWLEDGEMENTS}

We gratefully acknowledge Reija Randen for her excellent technical assistance. The study was supported financially by the Medical Research Fund of Tampere University Hospital.

Neal D, Sharples L, Smith K, Fennelly J, Hall R, Harris A (1990) The epidermal growth factor receptor and prognosis of bladder cancer. Cancer 65: $1619-1625$

Piyathilake C, Frost A, Manne U, Weiss H, Bell W, Heimburger D, Grizzle W (2002) Differential expression of growth factors in squamous cell carcinoma and precancerous lesions of the lung. Clin Cancer Res 8: $734-744$

Sargent ER, Gomella LG, Beldegrum, Linehan WM, Kasid A (1989) Epidermal growth factor receptor gene expression in normal human kidney and renal cell carcinoma. J Urol 142: $1364-1368$

Slamon DJ, Clark GM, Wong SG, Levin WJ, Ullrich A, Mc Guire WL (1987) Human breast cancer: correlation of relapse and survival with amplification of her-2/neu oncogene. Science 235: 177-182

Uhlman DL, Nguyen P, Manivel JC, Zhang G, Hagen K, Fraley E, Aeppli D, Niehans GA (1995) Epidermal growth factor receptor and transforming growth factor alpha expression in papillary and nonpapillary renal cell carcinoma: correlation with metastatic behavior and prognosis. Clin Cancer Res 8: $913-920$

UICC International Union Against Cancer (1997), In TNM Classification of Malignant Tumors, Sobin LH, Wittekind Ch (eds) New York: John Wiley \& Sons, Inc., Publication

Vlaykova T, Talve L, Hahka-Kemppinen M, Hernberg M, Muhonen T, Collan Y, Pyrhönen S (2002) Immunohistochemically detectable bcl-2 expression in metastatic melanoma: association with survival and treatment response. Oncology 62: 259-268

Yoshida K, Hosoya Y, Sumi S, Honda M, Moriguchi H, Yano M, Ueda Y (1997) Studies of the expression of epidermal growth factor receptor in human renal cell carcinoma: a comparison of immunohistochemical method versus ligand binding assay. Oncology 54: $220-225$ 